\title{
Characterization of new negative temperature coefficient thermistors based on $\mathrm{Zn}-\mathrm{Ni}-\mathrm{O}$ system
}

\author{
Xiang SUN ${ }^{a}$, Hong ZHANG ${ }^{a, b}$, Ya $\mathrm{LIU}^{a}$, Jia GUO ${ }^{a}$, Zhicheng $\mathrm{LI}^{a, b, *}$ \\ ${ }^{a}$ School of Materials Science and Engineering, Central South University, Changsha 410083, China \\ ${ }^{b}$ State Key Laboratory of Powder Metallurgy, Central South University, Changsha 410083, China
}

Received: July 07, 2016; Revised: August 02, 2016; Accepted: August 25, 2016

(C) The Author(s) 2016. This article is published with open access at Springerlink.com

\begin{abstract}
Y}_{2} \mathrm{O}_{3}$-doped $\mathrm{Zn}_{1-x} \mathrm{Ni}_{x} \mathrm{O}(x=0,0.3,0.4,0.5,0.6,0.7$, and 0.9$)$ powders were prepared by a wet chemical synthesis method, and the related ceramics were obtained by the traditional ceramic sintering technology. The phases and related electrical properties of the ceramics were investigated. The analysis of X-ray diffraction (XRD) indicates that the prepared ceramics with Ni substitution have a cubic crystalline structure. The resistance-temperature feature indicates that all the ceramics show a typical effect of negative temperature coefficient (NTC) of resistivity with the thermal constants between 3998 and $5464 \mathrm{~K}$, and have high cyclical stability in a temperature range from 25 to $300{ }^{\circ} \mathrm{C}$. The impedance analysis reveals that both grain effect and grain boundary effect contribute collectively to the NTC effect. The electron hopping and band conduction models are proposed for the grain (bulk) conduction, and the thermally activated charge carrier transport overcoming the energy barrier is suggested for the grain boundary conduction.
\end{abstract}

Keywords: $\mathrm{Zn}_{1-x} \mathrm{Ni}_{x} \mathrm{O}$; thermistors; microstructure; electrical properties; conduction mechanism

\section{Introduction}

Negative temperature coefficient (NTC) thermistors are widely used in industrial and domestic devices. At present, the NTC thermistors for the commercial applications are always the solid solutions based on the transition metal oxides and have the spinel structure, such as $\mathrm{Mn}-\mathrm{Ni}-\mathrm{O}, \mathrm{Ni}-\mathrm{Cu}-\mathrm{Mn}-\mathrm{O}$, and $\mathrm{Mn}-\mathrm{Co}-\mathrm{N}-\mathrm{O}$ systems $[1,2]$. In the $\mathrm{AB}_{2} \mathrm{O}_{4}$-type spinel structure, there are two sites available for the cations: a tetrahedral site, A-site, and an octahedral site, B-site. Generally, $\mathrm{Mn}^{3+}$ occupies predominantly the B-site while $\mathrm{Mn}^{2+}$ locates at the $\mathrm{A}$-site, and almost all $\mathrm{Ni}^{2+}$ goes to the $\mathrm{B}$-site. In the spinel manganite ceramics, the conduction is generally

* Corresponding author.

E-mail: zhchli@csu.edu.cn believed to propagate by an electron hopping mechanism taking place between the $\mathrm{Mn}^{3+}$ and $\mathrm{Mn}^{4+}$ cations locating at the B-sites in the octahedron induced by lattice vibrations $[3,4]$. The exact oxidation states of the Mn cations in the spinel structure closely depend on sintering conditions including oxygen partial pressure, sintering temperature, and procedure. So those ceramics typically exhibit a considerable difference in resistivity for a minor change of oxygen partial pressure during sintering or annealing $[5,6]$.

On the other hand, the structural relaxation of those kinds of NTC compounds limits their applications at temperatures below $200{ }^{\circ} \mathrm{C}$. In $\mathrm{Ni}_{x} \mathrm{Mn}_{3-x} \mathrm{O}_{4}$ ceramics, the lowest electrical resistivity can be obtained at $x=$ 0.66 , and the value is about $1900 \Omega \cdot \mathrm{cm}[7,8]$. In order to further decrease the resistivity, $\mathrm{Cu}$ is doped into nickel manganites, e.g., the resistivity of $\mathrm{Cu}_{0.2} \mathrm{Ni}_{0.5} \mathrm{Mn}_{2.3} \mathrm{O}_{4}$ 
decreases to $70 \Omega \cdot \mathrm{cm}$. However, the doping of $\mathrm{Cu}$ will cause a harmful consequence, i.e., the resistivity drift rate is $14.9 \%$ after ageing at $150{ }^{\circ} \mathrm{C}$ for $500 \mathrm{~h}$ in air [9]. For improving the ageing characteristic, much work has been done by cation doping and microstructure modification [9-11]. For instance, Ma et al. [12] reported that the resistivity drift for $\mathrm{Ni}_{0.6} \mathrm{Cu}_{0.5} \mathrm{Zn}_{x} \mathrm{Mn}_{1.9-x} \mathrm{O}_{4}$ after annealing at $150{ }^{\circ} \mathrm{C}$ for $500 \mathrm{~h}$ in air decreases sharply from $10.2 \%$ to $4.8 \%$ with $x$ increases from 0 to 0.25 .

For meeting the real applications, some new types of NTC thermistors have been developed, e.g., doped $\mathrm{Bi}_{2} \mathrm{O}_{3}$-based ceramics, inverse spinel type $\mathrm{Zn}_{7} \mathrm{Sb}_{2} \mathrm{O}_{12}$, pyrochlore type $\mathrm{Bi}_{3} \mathrm{Zn}_{2} \mathrm{Sb}_{3} \mathrm{O}_{14}$ compounds, and even modified simple oxides such as $\mathrm{SnO}_{2}$ and $\mathrm{CuO}$ [13-17]. One of the distinct advantages of the semiconductive oxides used for the NTC applications is that the room-temperature conductivity can be effectively adjusted by element doping and the related thermal-sensitive constants can also be improved by substituting suitable impurities [17-20].

Zinc oxide $(\mathrm{ZnO})$, as a semiconductor with the band gap of $3.37 \mathrm{eV}$, has attracted much attentions for several decades due to its potential applications in light emitting, solar cells, spintronic, transparent conducting, semiconductors, biosensors, and photocatalytic materials [21-24]. $\mathrm{NiO}$ is also one typical semiconductive material with the band gap of $3.6-4.0 \mathrm{eV}$ and has aroused numerous interests for various applications [25-28]. Recently, many reports on $\mathrm{Zn}-\mathrm{Ni}-\mathrm{O}$ materials have mainly focused on the crystalline structure, optical properties, and magnetic properties, etc. [29-31]. To the best of our knowledge, little work is focused on the temperature dependence of resistivity. During investigating the influence of $\mathrm{Ni}$ and $Y$ doping on the electrical property and crystal structure of the $\mathrm{ZnO}$ ceramics, the authors found that the $\mathrm{Zn}_{1-x} \mathrm{Ni}_{x} \mathrm{O}$ ceramics can not only transform from a hexagonal structure to a cubic one when the $\mathrm{Ni}$ concentration increases, but also show a perfect NTC effect. The temperature sensitivity of resistivity of the $\mathrm{Zn}_{1-x} \mathrm{Ni}_{x} \mathrm{O}(x=0.3,0.4,0.5,0.6,0.7$, and 0.9$)$ ceramics was investigated in the present work.

\section{Experimental procedures}

Ni-substituted $\mathrm{ZnO}, \mathrm{Zn}_{1-x} \mathrm{Ni}_{x} \mathrm{O}(x=0,0.3,0.4,0.5,0.6$,
0.7 , and 0.9 ) powders were prepared by a wet chemical process with polyvinyl alcohol as a polymeric carrier. In this work, $\mathrm{Y}_{2} \mathrm{O}_{3}$ was selected as the semiconducting dopant. The room-temperature resistivities of $\mathrm{Zn}_{1-x-y} \mathrm{Ni}_{x} \mathrm{Y}_{y} \mathrm{O}$ ceramics with different contents of $\mathrm{Y}_{2} \mathrm{O}_{3}$ $(y=0.005,0.01,0.015$, and 0.02) are in magnitude of $10^{3} \Omega \cdot \mathrm{cm}$; therefore, $y=0.01$ was used in the following experiments. Appropriate quantities of zinc oxide (analytical reagent), basic nickel carbonate (analytical reagent), and yttrium oxide (analytical reagent) were weighed according to the formula of $\mathrm{Zn}_{0.99-x} \mathrm{Ni}_{x} \mathrm{Y}_{0.01} \mathrm{O}$ $(x=0.3,0.4,0.5,0.6,0.7$, and 0.9 , denoted as ZNY3, ZNY4, ZNY5, ZNY6, ZNY7, and ZNY9, respectively). $\mathrm{ZnO}, \mathrm{NiCO}_{3} \cdot 2 \mathrm{Ni}(\mathrm{OH})_{2} \cdot 4 \mathrm{H}_{2} \mathrm{O}$, and $\mathrm{Y}_{2} \mathrm{O}_{3}$ were dissolved into nitric acid solution (about $5 \mathrm{vol} \% \mathrm{HNO}_{3}$ in distilled $\mathrm{H}_{2} \mathrm{O}$ ), respectively. Then the solutions were mixed together. A proper amount of polyvinyl alcohol (PVA), as the polymeric carrier for the effective preparation of mixed oxides, was added into the solution. The mixed solution was heated and dried under magnetic stirring to get the ZNY precursors. The ZNY powders were calcined at $1000{ }^{\circ} \mathrm{C}$ for $3 \mathrm{~h}$, and then were pressed into pellets of $16 \mathrm{~mm}$ in diameter and about $3 \mathrm{~mm}$ in thickness. The green pellets were sintered at $1350{ }^{\circ} \mathrm{C}$ for $2 \mathrm{~h}$ in air. The surfaces of sintered pellets (ceramics) were polished, and the electrodes were made on both opposite sides with silver paste by heating at $600{ }^{\circ} \mathrm{C}$ for about $5 \mathrm{~min}$.

The crystalline structure and phase component of the as-sintered samples were measured by X-ray diffraction (XRD, Rigaku D/max 2,500, Japan) with $\mathrm{Cu} \mathrm{Ka}$ radiation and $\lambda$ of $0.154056 \mathrm{~nm}$ in the diffraction angle $2 \theta$ between $5^{\circ}$ and $80^{\circ}$ at the scanning rate of $8\left(^{\circ}\right) / \mathrm{min}$. The ceramic microstructures were investigated with a scanning electron microscope (SEM, FEI Quanta200). For the SEM observations, the sintered ceramics were broken into pieces and the fracture surface morphology was observed. The valence state of the Ni-ion in the ceramics was investigated by X-ray photoelectron spectroscopy (XPS, K-alpha 1063, UK).

The temperature dependence of resistivity $(R-T)$ of the samples was tested in the temperature range from 25 to $300{ }^{\circ} \mathrm{C}$ by an $R-T$ measurement system (ZWX-C, Huazhong University of Science and Technology, China). For the property stability measurement, the samples were repeatedly tested from 25 to $300{ }^{\circ} \mathrm{C}$ by an 
$R-T$ measurement system; after each measurement, the samples were cooled with the stove. Alternating current (AC) impedance measurements were performed to analyze the conduction characteristic of the ceramics from 25 to $70{ }^{\circ} \mathrm{C}$ by an electrochemistry test system (Gamry Reference 600, USA), and the test frequency ranged from $1 \mathrm{~Hz}$ to $1 \mathrm{MHz}$.

\section{Results and discussion}

\section{1 Phase and microstructure}

Figure 1 shows the $\mathrm{XRD}$ patterns of the as-sintered $\mathrm{Zn}_{0.99-x} \mathrm{Ni}_{x} \mathrm{Y}_{0.01} \mathrm{O}(x=0,0.3,0.5$, and 0.7$)$ ceramics. The main diffraction peaks, for the ceramics $x=0.3,0.5$, and 0.7 , belong to the cubic phase with a space group of $F m \overline{3} m$ (referred by the PDF No. 75-0273). Beside these, one weak XRD peak can be detected at $2 \theta \approx 29.23^{\circ}$ as denoted by symbol " "; the weak XRD peak might be the one from $\mathrm{Y}_{2} \mathrm{O}_{3}$ (referred by the PDF No. 43-0661, space group of $F m \overline{3} \mathrm{~m}$, and lattice parameter of $a=$ $0.5264 \mathrm{~nm})$. By being refined with the Jade $6.0+$ pdf2004 program, the lattice parameters are 0.4224 , 0.4206 , and $0.4193 \mathrm{~nm}$ for the Ni contents of $x=0.3,0.5$, and 0.7 , respectively. While, the Y-doped $\mathrm{ZnO}$ $\left(\mathrm{Zn}_{0.99} \mathrm{Y}_{0.01} \mathrm{O}\right)$ ceramic has a hexagonal structure with space group of $P 6_{3} m c$. These indicate that $\mathrm{ZnO}$ crystal changes from hexagonal to cubic structure by substitution with $\mathrm{NiO}$.

The SEM micrograph obtained from the section of the as-sintered ZNY5 ceramic is shown in Fig. 2. The morphology of the fracture surface shows a polycrystalline characteristic with the mean grain size about $3 \mu \mathrm{m}$. The grains connect with each other closely

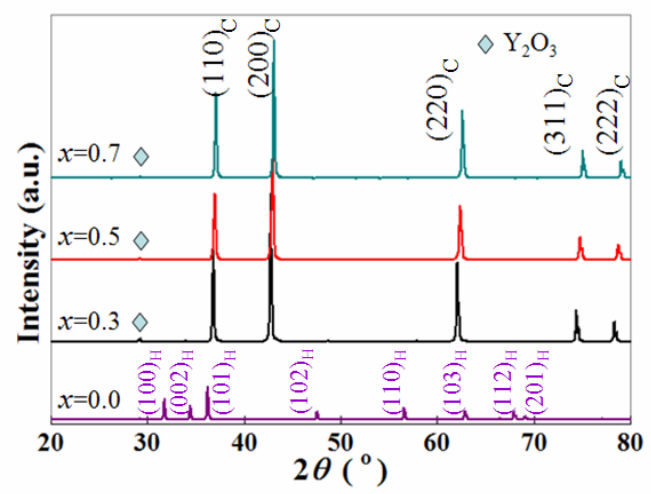

Fig. 1 XRD patterns of the as-sintered $\mathrm{Zn}_{0.99-x} \mathrm{Ni}_{x} \mathrm{Y}_{0.01} \mathrm{O}$ $(x=0,0.3,0.5,0.7)$ ceramics.

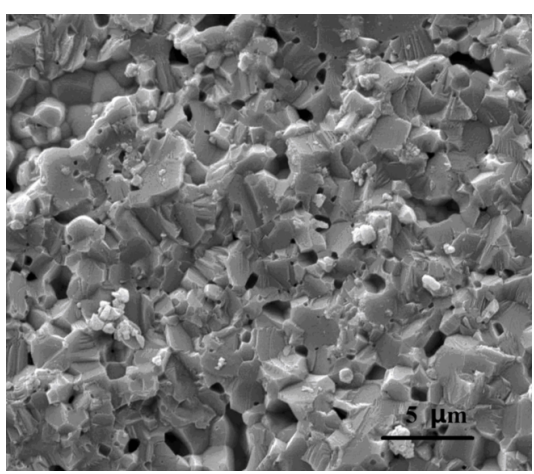

Fig. 2 SEM micrograph of the as-sintered ZNY5 ceramic.

although there are some pores in the ceramic.

\section{2 Electrical properties}

Figure 3(a) shows the Arrhenius plots of the temperature dependence of resistivity $(\ln \rho-1000 / T)$, measured by DC $R-T$ system, of the ZNY ceramics with various $\mathrm{Ni}$ contents. All the $\mathrm{ZNY}$ ceramics show the typical NTC effect. The resistivity $(\ln \rho)$ versus $1000 / T$ relationship in the testing temperature range from 25 to $300{ }^{\circ} \mathrm{C}$ is nearly linear. The resistivity-temperature relationship could be approximately expressed as Eq. (1):

$$
\rho_{T}=A \exp \frac{E_{\mathrm{a}}}{k T}=A \exp \frac{B}{T}
$$

where $\rho_{T}$ is the resistivity at temperature $T, T$ is the temperature in Kelvin, $k$ is the Boltzmann constant, $A$ is a constant related to the material characteristic, $E_{\mathrm{a}}$ is the activation energy of conduction, and $B$ is the NTC thermal constant which is usually applied to characterize the temperature sensitivity of an NTC thermistor. In the real applications, the NTC $B$ value is always calculated by using the data measured at 25 and $85^{\circ} \mathrm{C}$ by Eq. (2):

$$
B_{25 / 85}=\frac{\ln \rho_{25}-\ln \rho_{85}}{1 / T_{25}-1 / T_{85}}
$$

where $\rho_{25}$ and $\rho_{85}$ are the resistivities at 25 and $85{ }^{\circ} \mathrm{C}$, respectively.

The Ni-concentration dependence of $\rho_{25}$ and $B_{25 / 85}$ of the ZNY ceramics are shown in Fig. 3(b). It can be seen that both $\rho_{25}$ and $B_{25 / 85}$ can be effectively adjusted by changing the $\mathrm{Ni}$ concentration. For the concentrations $x$ are $0.3,0.4,0.5,0.6,0.7$, and $0.9, \rho_{25}$ of the ZNY ceramics are 20.4, 11.3, 4.84, 3.61, 4.0, and $0.637 \mathrm{k} \Omega \cdot \mathrm{cm}^{-1}$, respectively, and the thermal constant $B$ values are calculated to be $4782,5054,5464,5366$, 

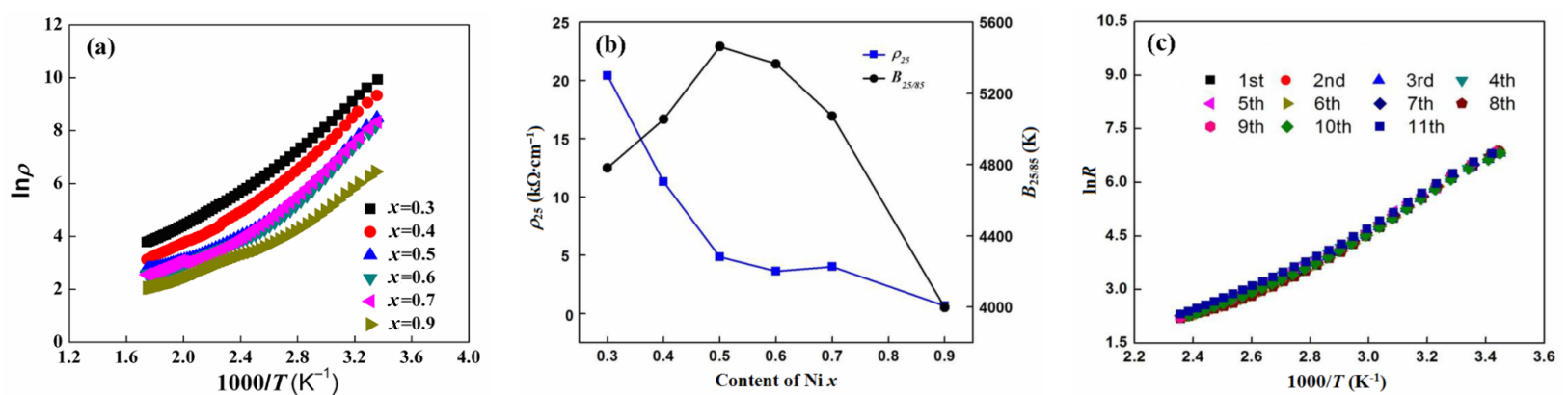

Fig. 3 Electrical properties of the ceramics: (a) temperature dependence of resistivity of the $\mathrm{Zn}_{0.99-x} \mathrm{Ni}_{x} \mathrm{Y}_{0.01} \mathrm{O}(x=0.3,0.4,0.5,0.6$, $0.7,0.9$ ) ceramics in Arrhenius plots; (b) Ni-concentration dependence of $\rho_{25}$ and $B_{25 / 85}$; (c) $R-T$ plots of a ZNY ceramic repeatedly measured for 11 times, showing high cyclic stability.

5072, and $3998 \mathrm{~K}$, respectively. For the commercial applications, $B$ values are required to be $2000-6000 \mathrm{~K}$, and are always about $4000 \mathrm{~K}$ in fact. The adjustable $\rho_{25}$ and $B_{25 / 85}$ of the ZNY ceramics indicate that the ZNY system thermistors have great potential for practical applications.

To test the property stability of the ZNY thermistors, repeated $R-T$ measurement of a ZNY5 sample was performed. Figure 3(c) shows the temperature dependence of resistivity repeatedly tested from 25 to $300{ }^{\circ} \mathrm{C}$ for 11 times. The $R-T$ plots of ZNY5 ceramic exhibit a centralized data distribution and nearly repeatable NTC behavior, indicating a high cyclic stability.

\section{3 Impedance and electric modulus analysis}

In order to further understand the conduction characteristic of the ZNY ceramics, AC impedance spectroscopy was employed here. The impedance data $Z(\omega)$ is usually plotted in the Cole-Cole plane as Eq. (3):

$$
Z(\omega)=Z^{\prime}+\mathrm{j} Z^{\prime \prime}
$$

where $\omega$ is the angular frequency, $Z^{\prime}$ and $Z^{\prime \prime}$ are the real and imaginary parts of the complex impedance respectively, and $\mathrm{j}=\sqrt{-1}$ is the imaginary factor. In general, a complex impedance spectrum of an ideal polycrystalline ceramic is usually made up of three semicircles, which represent grain effect, grain boundary effect, and the electrode polarization effect [32].

Figure 4 shows the Nyquist plots of complex impedance spectra of ZNY3 ceramic at 25, 40, 55, and $70{ }^{\circ} \mathrm{C}$. All the plots show part of arcs of the impedance spectra. For the limited frequency of the measurement system, it could not be obtained the ideal spectra that consist of typical semicircles. An equivalent circuit is applied to fit the measured impedance data (see the inset in Fig. 4). Here, $R 1$ and $R 2$ represent the resistances from grain effect and grain boundary effect, respectively; $C P E 1$ and $C P E 2$ are the constant phase elements for the effect due to interior structure inhomogeneity or some other uncertain defects in the material. The impedance response of a constant phase element (CPE) can be defined as $Z_{\mathrm{CPE}}=1 /\left[(\mathrm{j} \omega)^{\alpha} Q\right]$ [33]. Here, $Q$ and $\alpha$ are the CPE parameters which are frequency independent. The parameter $\alpha$ mathematically represents an angle of rotation in the complex plane with respect to the impedance response of an ideal capacitor, and it always ranges between 0.5 and 1. The CPE coefficient $Q$ is the combination of properties related to both the surface and the electro-active species, and represents the differential capacitance of the interface when $\alpha=1$. As shown in Fig. 4 , the fitted curves match the measured data well, revealing that there are two arcs reflecting grain effect

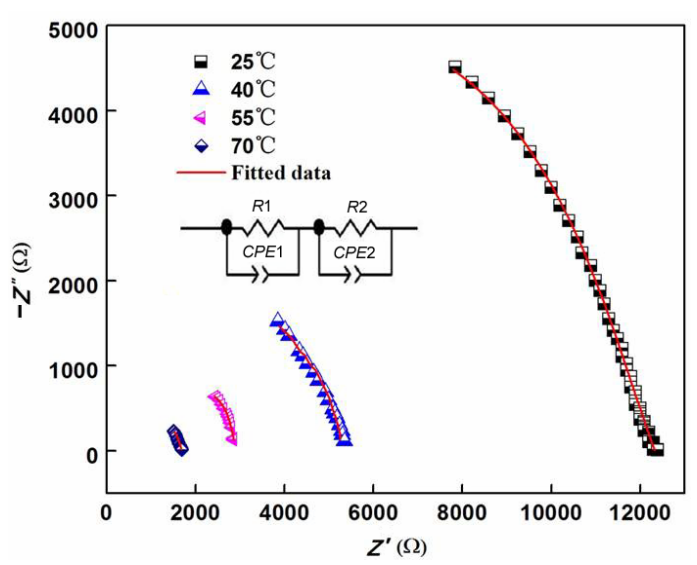

Fig. 4 Analysis of Nyquist plots of ZNY3 ceramic measured at $25,40,55$, and $70{ }^{\circ} \mathrm{C}$ (the inset equivalent circuit is used for fitting the impedance data). 
and grain boundary effect in each impedance spectrum, and grain effect in the higher frequency part and grain boundary effect in the lower frequency part overlap with each other. However, the electrode polarization effect is too weak to be detected.

By using $\sigma(\omega)=1 / Z(\omega)$, the complex impedance $Z(\omega)$ can be converted into a complex admittance $\sigma(\omega)$. The AC conductivity $\sigma^{\prime}(\omega)$ obeys the Jonscher power law as given by Eq. (4) [34]:

$$
\sigma^{\prime}(\omega)=\sigma^{\prime}(0)+A \omega^{S}
$$

where $\sigma^{\prime}(\omega) \quad\left(\sigma^{\prime}(\omega)=Z^{\prime} /|Z|^{2}\right)$ is the real part conductivity depending on frequency, $\omega$ is the angular frequency, $\sigma^{\prime}(0)$ is the DC conductivity which can be extracted from the value of $\sigma^{\prime}(\omega)$ when $\omega$ tends to zero; $S$ is the power exponent, which is temperature and material dependent, but not frequency independent, and it is considered to be less than 1 for hopping conduction [35].

Figure 5 shows the double-logarithmic plots of electrical conductivity versus frequency, $\log \omega$, of the ZNY3 ceramic at various temperatures. For a certain frequency, the conductivity $\sigma^{\prime}(\omega)$ increases with the rise in temperature. While, $\log \sigma^{\prime}(\omega)$ keeps almost unchangeable in the low frequency region, but becomes frequency sensitive in the high frequency region. $S$ values are obtained to be always less than 1 , by derived from the slope of the plots, and tend to decrease with the increasing temperature. These suggest that the electron hopping conduction is one of the conduction models in the ZNY thermistors, which will be further discussed in the next section.

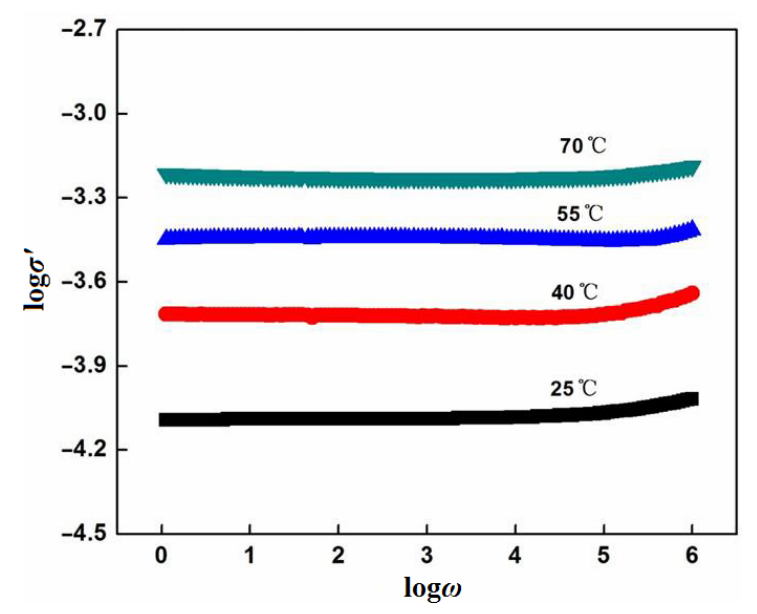

Fig. 5 Double-logarithmic plots $\left(\log \sigma^{\prime}(\omega)-\log \omega\right)$ for the $\mathrm{ZNY} 3$ ceramic at various temperatures.

\section{4 Conduction mechanisms}

In the spinel manganite ceramics, the conduction is generally believed to propagate by an electron hopping mechanism taking place between the $\mathrm{Mn}^{3+}$ and $\mathrm{Mn}^{4+}$ cations locating at the B-sites in the octahedron induced by lattice vibrations $[3,4]$. The related conduction mechanism is so-called electron hopping model. The valence states of the cations always depend on the sintering conditions; therefore, the room-temperature resistivities of the spinel manganite ceramics can not be artificially adjusted as that of the traditional semiconductor whose conduction is based on the energy band theory.

Both $\mathrm{NiO}$ and $\mathrm{ZnO}$ are semiconductors with the band gaps of $3.37 \mathrm{eV}$ and 3.6-4.0 eV, respectively. The solid solutions of $\mathrm{Zn}_{1-x} \mathrm{Ni}_{x} \mathrm{O}$ are also semiconductors and the related band gap changes with the $\mathrm{Zn} / \mathrm{Ni}$ ionic ratio. For example, as calculated by Mi et al. [36], a band gap of $3.25 \mathrm{eV}$ was in the band structure of the majority spins and $2.60 \mathrm{eV}$ in the band structure of minority spins in $\mathrm{Ni}_{0.5} \mathrm{Zn}_{0.5} \mathrm{O}$. In the mean time, it is well known that $\mathrm{Y}$ substitution in ZNY ceramics acts as the donor doping and occurs a donor energy level near the conduction level. The related defect reaction can be written as Eq. (5):

$$
\mathrm{Y}_{2} \mathrm{O}_{3} \stackrel{\mathrm{Zn}_{1-x} \mathrm{Ni}_{x} \mathrm{O}}{\longrightarrow} \mathrm{Y}_{\mathrm{Zn}(\mathrm{Ni})}^{\cdot}+2 \mathrm{O}_{\mathrm{O}}^{\times}+\frac{1}{2} \mathrm{O}_{2}+2 \mathrm{e}^{\prime}
$$

As a result, the electrons at the donor energy level can be easily activated to transport to the conduction level and contribute the conduction of the ZNY ceramics. This is the so-called band conduction mechanism. As discussed in Fig. 4, each impedance spectrum is composed of grain effect (bulk effect) and grain boundary effect. The band conduction mechanism should be one of the possible conduction models for the bulk effect in the ones for the ZNY ceramic thermistors. For a donor doped n-type semiconductor, the conductivity $\sigma$ can be described as Eq. (6):

$$
\sigma=N \exp \left(-\frac{E_{\mathrm{c}}-E_{\mathrm{d}}}{2 k T}\right) e \mu
$$

where $N$ is the effective state density of conduction band, $E_{\mathrm{c}}$ and $E_{\mathrm{d}}$ are respectively the conduction band bottom level and donor level, $e$ is the charge of conducting electron, and $\mu$ is the electronic mobility which depends on the lattice structure and temperature. One can see that the conductivity shows exponential relationship with the temperature, indicating that the resistivity also has an exponential relationship with the 
temperature, i.e., typical NTC effect.

On the other hand, as discussed in Eq. (4) and Fig. 5, the power exponent $S$ is always less than 1, indicating that the electron hopping conduction should be another conduction model for the ZNY conduction. For the hopping conduction, there must be valence-variable ions which have different valences locating at the same crystallographic site [37]. Generally, Ni-ion might have different valences for the arrangement of extra-nuclear electron of $3 d^{8} 4 s^{2}$, but Zn-ion and Y-ion do not have variable valence. To distinguish the possible valences of the Ni-ions in the ZNY ceramics, XPS analysis of ZNY 5 ceramic was performed as shown in Fig. 6. One can see from the XPS spectrum that, the Ni $2 \mathrm{p}_{3 / 2}$ peak and its satellite one show the binding energies of 853.4 and $860.2 \mathrm{eV}$, respectively; and the $\mathrm{Ni} 2 \mathrm{p}_{1 / 2}$ main peak and its satellite one locate at 871.6 and $878.5 \mathrm{eV}$, respectively. These are in accordance with that reported by Gokul et al. [38]. Because of the existence of $Y$ and $\mathrm{Zn}$ ions, the binding energies show a small shift towards lower one, comparing to the pure NiO [38-40]. This indicates that two kinds of valences of $\mathrm{Ni}^{3+}$ oxidation state and $\mathrm{Ni}^{2+}$ oxidation state exist in the ZNY ceramics. This provides the possibility for the hopping conduction. The electron hopping between $\mathrm{Ni}^{3+}$ and $\mathrm{Ni}^{2+}$ ionic pairs can be expressed as $\mathrm{Ni}^{3+}+\mathrm{Ni}^{2+} \leftrightarrow \mathrm{Ni}^{2+}+\mathrm{Ni}^{3+}$. When the temperature increases, the thermal activation increases and enhances the electron hopping, resulting in the NTC effect.

Because of the hopping conduction and the band conduction, the resistivity of grain effect decreases obviously with the temperature increases, indicating the NTC effect.

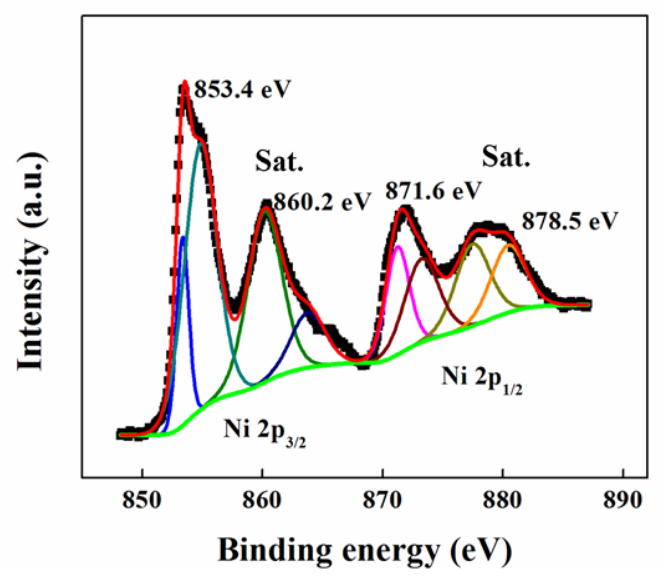

Fig. 6 XPS spectrum of the Ni 2p in the ZNY5 ceramic.
For the resistivity from the grain boundary effect, the grain boundary barrier should be the main obstacle for the electron transport. Because the grain boundary has complicate composition and structure, the related conduction mechanism should not simply follow the ones inside the grain. The charge carriers (electron and/or electronic hole) can transport between grains by overcoming the barrier energy by thermal activation. Assuming that the barrier energy is $E_{\mathrm{b}}$, the probability for a charge carrier reaching $E_{\mathrm{b}}$ is proportional to $\exp \left(-E_{\mathrm{b}} / k T\right)$, according to the Boltzmann statistical distribution law. Here, $k$ is the Boltzmann constant and $T$ is the temperature in Kelvin. This indicates that the transport chance for the charge carrier overcoming the grain boundary barrier increases is in exponential relation with the temperature rise, i.e., the resistivity from the grain boundary effect shows NTC characteristic induced by thermal activation.

\section{Conclusions}

$\mathrm{Zn}_{0.99-x} \mathrm{Ni}_{x} \mathrm{Y}_{0.01} \mathrm{O}(x=0.3,0.4,0.5,0.6,0.7$, and 0.9$)$ with cubic crystalline structure were prepared by a wet chemical synthesis method followed by the traditional ceramic sintering technology. $\mathrm{Zn}_{0.99-x} \mathrm{Ni}_{x} \mathrm{Y}_{0.01} \mathrm{O}$ ceramics have a novel NTC effect with the thermal constants between 3998 and $5464 \mathrm{~K}$, and have a high temperature cyclic stability between 25 and $300{ }^{\circ} \mathrm{C}$. Both grain effect and grain boundary effect contribute to the resistivity NTC effect of the ceramics. Hopping conduction such as $\mathrm{Ni}^{3+}+\mathrm{Ni}^{2+} \leftrightarrow \mathrm{Ni}^{2+}+\mathrm{Ni}^{3+}$ and band conduction are proposed to be the conduction mechanism for bulk conduction effect, and thermal activation transport of charge carriers is proposed for the conduction mechanism for grain boundary effect.

\section{Acknowledgements}

The authors acknowledge the financial support provided by the National Natural Science Foundation of China (No. 51172287) and the Laboratory Research Fund by the State Key Laboratory of Powder Metallurgy, Central South University, China.

\section{References}

[1] Gao H, Ma C, Sun B. Preparation and characterization of 
$\mathrm{NiMn}_{2} \mathrm{O}_{4}$ negative temperature coefficient ceramics by solid-state coordination reaction. J Mater Sci: Mater Electron 2014, 25: 3990-3995.

[2] Jadhav RN, Mathad SN, Puri V. Studies on the properties of $\mathrm{Ni}_{0.6} \mathrm{Cu}_{0.4} \mathrm{Mn}_{2} \mathrm{O}_{4} \mathrm{NTC}$ ceramic due to Fe doping. Ceram Int 2012, 38: 5181-5188.

[3] Macklen ED. Electrical conductivity and cation distribution in nickel manganite. J Phys Chem Solids 1986, 47: 1073-1079.

[4] Jung J, Töpfer J, Mürbe J, et al. Microstructure and phase development in $\mathrm{NiMn}_{2} \mathrm{O}_{4}$ spinel ceramics during isothermal sintering. J Eur Ceram Soc 1990, 6: 351-359.

[5] Fau P, Bonino JP, Demai JJ, et al. Thin films of nickel manganese oxide for NTC thermistor applications. Appl Surf Sci 1993, 65: 319-324.

[6] Basu A, Brinkman AW, Schmidt R. Effect of oxygen partial pressure on the NTCR characteristics of sputtered $\mathrm{Ni}_{x} \mathrm{Mn}_{3-x} \mathrm{O}_{4+\delta}$ thin films. $J$ Eur Ceram Soc 2004, 24: 1247-1250.

[7] Fritsch S, Sarrias J, Brieu M, et al. Correlation between the structure, the microstructure and the electrical properties of nickel manganite negative temperature coefficient (NTC) thermistors. Solid State Ionics 1998, 109: 229-237.

[8] Metz R. Electrical properties of N.T.C. thermistors made of manganite ceramics of general spinel structure: $\mathrm{Mn}_{3-x-x^{\prime}} \mathrm{M}_{x} \mathrm{~N}_{x} \mathrm{O}_{4}\left(0 \leqslant x+x^{\prime} \leqslant 1 ; \mathrm{M}\right.$ and $\mathrm{N}$ being Ni, Co or $\mathrm{Cu}$ ). Aging phenomenon study. J Mater Sci 2000, 35: 4705-4711.

[9] Zhao C, Wang B, Yang P, et al. Effects of $\mathrm{Cu}$ and $\mathrm{Zn}$ co-doping on the electrical properties of $\mathrm{Ni}_{0.5} \mathrm{Mn}_{2.5} \mathrm{O}_{4} \mathrm{NTC}$ ceramics. J Eur Ceram Soc 2008, 28: 35-40.

[10] Tadokoro SK, Muccillo ENS. Effect of Y and Dy co-doping on electrical conductivity of ceria ceramics. J Eur Ceram Soc 2007, 27: 4261-4264.

[11] Park K, Lee JK, Kim S-J, et al. The effect of Zn on the microstructure and electrical properties of $\mathrm{Mn}_{1.17-x} \mathrm{Ni}_{0.93} \mathrm{Co}_{0.9} \mathrm{Zn}_{x} \mathrm{O}_{4}(0 \leqslant x \leqslant 0.075)$ NTC thermistors. J Alloys Compd 2009, 467: 310-316.

[12] Ma C, Liu Y, Lu Y, et al. Effect of Zn substitution on the phase, microstructure and electrical properties of $\mathrm{Ni}_{0.6} \mathrm{Cu}_{0.5} \mathrm{Zn}_{x} \mathrm{Mn}_{1.9-x} \mathrm{O}_{4}(0 \leqslant x \leqslant 1)$ NTC ceramics. Mat Sci Eng B 2014, 188: 66-71.

[13] Sahoo S, Parashar SKS, Ali SM. $\mathrm{CaTiO}_{3}$ nano ceramic for NTCR thermistor based sensor application. $J$ Adv Ceram 2014, 3: 117-124.

[14] Basu A, Brinkman AW, Hashemi T. NTC characteristics of bismuth based ceramic at high temperature. Int $J$ Inorg Mater 2001, 3: 1219-1221.

[15] Nobre MAL, Lanfredi S. Thermistor ceramic with negative temperature coefficient based on $\mathrm{Zn}_{7} \mathrm{Sb}_{2} \mathrm{O}_{12}$ : An inverse spinel-type phase. Appl Phys Lett 2002, 81: 451-453.

[16] Nobre MAL, Lanfredi S. Negative temperature coefficient thermistor based on $\mathrm{Bi}_{3} \mathrm{Zn}_{2} \mathrm{Sb}_{3} \mathrm{O}_{14}$ ceramic: An oxide semiconductor at high temperature. Appl Phys Lett 2003, 82: 2284-2286.

[17] Yang B, Zhang H, Zhang J, et al. Electrical properties and temperature sensitivity of B-substituted CuO-based ceramics for negative temperature coefficient thermistors.
J Mater Sci: Mater Electron 2015, 26: 10151-10158.

[18] Ouyang P, Zhang H, Zhang Y, et al. Zr-substituted $\mathrm{SnO}_{2}$-based NTC thermistors with wide application temperature range and high property stability. J Mater Sci: Mater Electron 2015, 26: 6163-6169.

[19] Zhang Y, Wu Y, Zhang $\mathrm{H}$, et al. Characterization of negative temperature coefficient of resistivity in $\left(\mathrm{Sn}_{1-x} \mathrm{Ti}_{x}\right)_{0.95} \mathrm{Sb}_{0.05} \mathrm{O}_{2}(x \leqslant 0.1)$ ceramics. J Mater Sci: Mater Electron 2014, 25: 5552-5559.

[20] Zhang J, Zhang H, Yang B, et al. Temperature sensitivity of Fe-substituted $\mathrm{SnO}_{2}$-based ceramics as negative temperature coefficient thermistors. J Mater Sci: Mater Electron 2016, 27: 4935-4942.

[21] Jiang F, Peng Z, Zang Y, et al. Progress on rare-earth doped ZnO-based varistor materials. J Adv Ceram 2013, 2: 201-212.

[22] Jaffe JE, Pandey R, Kunz AB. Electronic structure of the rocksalt-structure semiconductors $\mathrm{ZnO}$ and CdO. Phys Rev B 1991, 43: 14030-14034.

[23] Chen Y, Reyes PI, Duan Z, et al. Multifunctional ZnO-based thin-film bulk acoustic resonator for biosensors. J Electron Mater 2009, 38: 1605-1611.

[24] Lietti L, Tronconi E, Forzatti P. Surface properties of ZnO-based catalysts and related mechanistic features of the higher alcohol synthesis by FT-IR spectroscopy and TPSR. J Mol Catal 1989, 55: 43-54.

[25] Du Y, Wang W, Li X, et al. Preparation of $\mathrm{NiO}$ nanoparticles in microemulsion and its gas sensing performance. Mater Lett 2012, 68: 168-170.

[26] Zhang F-b, Zhou Y-k, Li H-l. Nanocrystalline NiO as an electrode material for electrochemical capacitor. Mater Chem Phys 2004, 83: 260-264.

[27] Wang X, Li X, Sun X, et al. Nanostructured NiO electrode for high rate Li-ion batteries. $J$ Mater Chem 2011, 21: 3571-3573.

[28] Ma Z, Zhang H, Zhang Y, et al. Electrochemical characteristics of nanostructured $\mathrm{NiO}$ plates hydrothermally treated on nickel foam for Li-ion storage. Electrochim Acta 2015, 176: 1427-1433.

[29] Dubinin SF, Maksimov VI, Parkhomenko VD, et al. Fine structure and magnetism of the cubic oxide compound $\mathrm{Ni}_{0.3} \mathrm{Zn}_{0.7} \mathrm{O}$. Phys Solid State 2011, 53: 1362-1366.

[30] Zuo Y, Ge S, Chen Z, et al. Morphology, optical and magnetic properties of $\mathrm{Zn}_{1-x} \mathrm{Ni}_{x} \mathrm{O}$ nanorod arrays fabricated by hydrothermal method. $J$ Alloys Compd 2009, 470: 47-50.

[31] Liu Z, Zhang Q, Shi G, et al. Solvothermal synthesis and magneto-optical properties of $\mathrm{Zn}_{1-x} \mathrm{Ni}_{x} \mathrm{O}$ hierarchical microspheres. J Magn Magn Mater 2011, 323: 1022-1026.

[32] Ouyang P, Zhang H, Xue D, et al. NTC characteristic of $\mathrm{SnSb}_{0.05} \mathrm{O}_{2}-\mathrm{BaTi}_{0.8} \mathrm{Fe}_{0.2} \mathrm{O}_{3-\delta}$ composite materials. J Mater Sci: Mater Electron 2013, 24: 3932-3939.

[33] Dominguez-Benetton X, Sevda S, Vanbroekhoven K, et al. The accurate use of impedance analysis for the study of microbial electrochemical systems. Chem Soc Rev 2012, 41: 7228-7246.

[34] Bruce PG. High and low frequency Jonscher behaviour of an ionically conducting glass. Solid State Ionics 1985, 15: 
247-251.

[35] Sayer M, Mansingh A, Reyes JM, et al. Polaronic hopping conduction in vanadium phosphate glasses. $J$ Appl Phys 1971, 42: 2857-2864.

[36] Mi W, Yang H, Cheng Y, et al. Ferromagnetic half-metallic characteristic in bulk $\mathrm{Ni}_{0.5} M_{0.5} \mathrm{O}(M=\mathrm{Cu}, \mathrm{Zn}$ and $\mathrm{Cd})$ : A GGA+U study. Solid State Commun 2012, 152: 1108-1111.

[37] Xue D, Zhang H, Li Y, et al. Electrical properties of hexagonal $\mathrm{BaTi}_{1-x} \mathrm{Fe}_{x} \mathrm{O}_{3-\delta}(x=0.1,0.2,0.3)$ ceramics with NTC effect. J Mater Sci: Mater Electron 2012, 23: 1306-1312.

[38] Gokul B, Saravanan P, Vinod VTP, et al. A study on the origin of room temperature ferromagnetism in $\mathrm{Ni}_{1-x} \mathrm{Gd}_{x} \mathrm{O}$ nanoparticles. J Magn Magn Mater 2015, 394: 179-184.

[39] Thema FT, Manikandan E, Gurib-Fakim A, et al. Single phase Bunsenite $\mathrm{NiO}$ nanoparticles green synthesis by Agathosma betulina natural extract. J Alloys Compd 2016, 657: 655-661.

[40] Subalakshmi P, Sivashanmugam A. Fuel aided synthesis of $\mathrm{NiO}$ flakes for electrochemical energy storage application. J Alloys Compd 2016, 662: 200-207.

Open Access The articles published in this journal are distributed under the terms of the Creative Commons Attribution 4.0 International License (http://creativecommons. org/licenses/by/4.0/), which permits unrestricted use, distribution, and reproduction in any medium, provided you give appropriate credit to the original author(s) and the source, provide a link to the Creative Commons license, and indicate if changes were made. 